\title{
LOCATION UPDATION FOR ENERGY EFFICIENT GEOGRAPHIC ROUTING IN
}

\author{
MANET \\ B.Manimegalai ${ }^{1}$, D.Geetha ${ }^{2}$ \\ ${ }^{1}$ Computer Science and Engineering, Adhiyamaan college of engineering, TamilNadu,India,bmaniit17@gmail.com \\ ${ }^{2}$ Computer Science and Engineering, Adhiyamaan college of Engineering, Tamil Nadu,India,dgeetha78@yahoo.com
}

\begin{abstract}
Routing in mobile ad-hoc networks with a large number of nodes or with high mobility is a very difficult task and energy conservation is very important for mobile devices. In geographic routing each node require information about neighbors to forward data packets. Earlier periodic updations are used for location updation but it consumes much amount of node energy and bandwidth utilized for unnecessary updation where there is no changes in the location information. In this paper adaptive position update used with less energy consumption and utilize the bandwidth for location updation only when there is a change in the network. The GPSR protocol used for packet forwarding in both greedy forwarding and perimeter forwarding. This adaptive updation utilize only less amount of node energy than other beaconing scheme.
\end{abstract}

Index Terms:Routing protocols, Beacon updation scheme, Wireless communication, Greedy forwarding, Adaptive position updation.

\section{INTRODUCTION}

With the popularity of positioning devices (e.g. GPS) and other localization schemes [1] geographic routing protocols are becoming an attractive choice for use in mobile ad hoc networks. The underlying principle used in these protocols involves selecting the next routing hop from among a node's neighbors, which is geographically closest to the destination [1]. It is necessary to consider not only the cost of transmitting a packet, but also of receiving, and even of discarding it [12]. Reduced data transmit and receive times have only limited impact on per-packet energy consumption, due to the height fixed overhead. Ad hoc mode operation incurs an extremely high idle cost compared to operation in conjunction with a base station. ROME [11] demonstrating its effectiveness through extensive simulations especially geared to show that it is able to effectively support mobility. Due to the mobility of the nodes the topology of the network may change frequently and in unpredictable ways [6]. Every node is aware of its own position and is notified of its neighbors position through beacons [9], small packets broadcasted by the neighbors to announce their position.

A distributed mobility-management scheme with a class of uniform quorum systems (UQS) [6] is proposed for ad hoc networks. In UQS location databases are stored in the nodes themselves. This databases are dynamically organized into quorums, every two of which intersect at a constant number of databases. Compared with a conventional scheme with fixed associations, this scheme is more suitable for ad hoc network. GLS [5] is a new distributed location service which tracks mobile node locations. GLS combined with geographic forwarding allows the construction of ad hoc mobile networks that scale to a larger number of nodes than possible with previous work.
GLS is decentralized and runs on the mobile nodes themselves, requiring no fixed infrastructure. Each mobile node periodically updates a small set of other nodes (its location servers) with its current location. A node sends its position updates to its location servers without knowing their actual identities, assisted by a predefined ordering of node identifiers and a predefined geographic hierarchy. Routing algorithm is quite suitable for a dynamic selfstarting network [4] as required by users wishing to utilize ad hoc networks AODV provides loop free routes even while repairing broken links. Protocol complexity of DREAM does not appear to provide benefits over a flooding protocol [3]. Promiscuous mode operation improves the performance of DSR significantly [3]. Adding location information to DSR increases both the network load and the data packet delivery ratio. Location- Aided Routing (LAR) protocols limit the search for a new route to a smaller request zone of the ad hoc network. This results in a significant reduction in the number of routing messages. Location based routing is difficult when there areholes in the network connection and nodes are mobile so thattopology dynamically changed. Terminode routing uses acombination of location based routing, used when thedestination is far and link state routing is used when the destination is close.

GPSR makes greedy forwarding decisions [1] using only information about a router's immediate neighbors in the network topology. When a packet reaches a region where greedy forwarding is impossible, the algorithm recovers by routing around the perimeter of the region. By keeping state only about the local topology, GPSR scales better in per router state than shortest path and ad hoc routing protocols as the number of network destinations increases. NADV [12] select neighbors with the optimal trade-off between 
proximity and link cost. Coupled with the local next hop decision in geographic routing, NADV provides an adaptive and efficient cost aware routing strategy. Depending on the objective or message priority, applications can use the NADV framework to minimize link cost.

\section{RELATED WORK}

Beacon less routing [10], [11] does not need any location information but it has high control packet overhead i.e. it needs to transfer control message before data transfer, so that it consumes high energy. Location updation is important in MANET because the node will not remain static. Periodic updation [11] cause high packet overhead so that we need a location updation scheme that has less packet overhead. Adaptive beaconing scheme has less packet overhead for location updation also it consumes less energy than periodic updation scheme.

\section{ENERGY CONSERVATION BY AD HOC NETWORK WITH LOCATION UPDATION}

- Energy needed for transmitting, receiving and discarding packets

In an efficient geographic routing node need to forward data packets only to the node that is closer to the destination but during broadcasting of data packets all the nodes in the network will receive the data packets so that number of packets transmitted during broadcasting is high when compared to point to point packet transmission. While comparing the energy needed for point to point data packet transmission with broadcasting, point to point consumes less energy. In broadcasting the node that is not a destination need to discard the packets another disadvantage of broadcasting is that it cause some security problem if any malicious node present in the network. So we need to use point to point forward of data packet transmission for energy efficient data transmission.

We use GPSR protocol [1] that forward packets only to neighbors that is closer to the destination. For example consider Fig. 1 with node $\mathrm{x}$ and neighbors A, B, C, D it will choose the neighbor $\mathrm{C}$ to reach $\mathrm{Y}$.

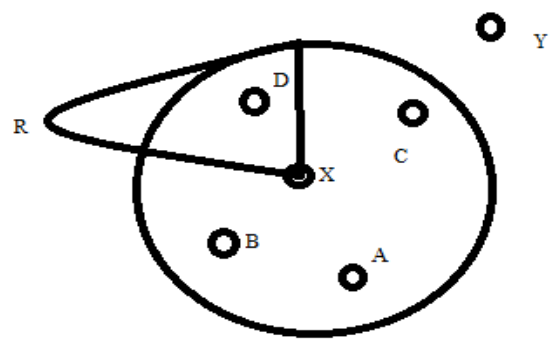

Fig-1: Node $X$ with transmission range of radius $R$ and neighbors A, B, C, D and destination Y.

- Energy needed for idle mode for a node
In ad hoc network nodes in idle mode before transmitting or receiving data packets, it consumes less energy than nodes periodically goes to a sleep or active state. For periodic sleep or active state periodic updation is more suitable with high packet delivery ratio and less energy consumption, but in idle mode ad hoc network the periodic updation cause high energy consumption i.e. utilize the bandwidth unnecessarily by updating location information about node where there is no change in the network nodes location. So we use APU location updation strategy [8] that utilize less energy consumption than periodic updation.

\section{- $\quad$ Energy needed for location updation packets}

For location updation a small control packet used to inform about the location change to other nodes in the network. Even small packet transmission also consumes node energy so that we need to reduce number of control packets needed for location updation. The APU strategy [8] only transmit beaconpackets to their neighbor instead of the entire network andonly transmit beacons whenever there is any change in thenetwork. The adaptive position update strategy consumes less energy and cause less beacon overhead than periodic updation in ad hoc network.

\section{RESULT}

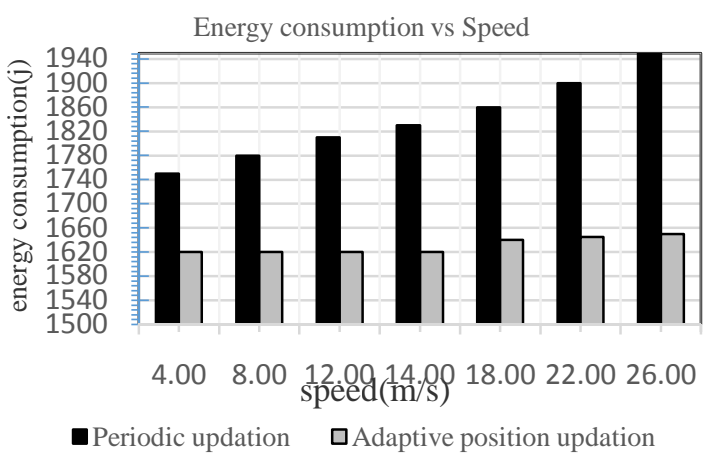

Fig-2: Energy consumption of two beaconing scheme with speed

The result shown in the Fig.2 clearly shows that energy consumption by adaptive updation scheme consumes less energy when compared to the periodic updation [8]. In Adaptive updation scheme the energy consumption increases as the speed increases but it is very less when compare to the periodic updation. This results are taken from the analysis of energy consumption of two beaconing scheme.

\section{CONCLUSION AND FUTURE WORK}

Energy efficiency is important for small and wireless devices. Location updation packet also consumes some amount of node energy so we need to reduce the number of packets needed for location updation. Adaptive updation scheme with GPSR protocol reduce the number of location updation packet, so that the node energy consumption is 
reduced when comparing to the periodic updation scheme. In our future work we decide to increase packet delivery ratio by combining periodic updation with adaptive updation scheme.

\section{REFERENCES}

[1]. B. Karp and H. T. Kung. "GPSR: Greedy Perimeter Stateless Routing for Wireless Networks", in Proceedings of ACM Mobicom2000, pp. 243-254, Boston, MA, USA, August 2000.

[2]. Y. Ko and N. H. Vaidya. "Location-Aided Routing (LAR) in Mobile Ad Hoc Networks", in ACM/BaltzerWireless Networks, vol. 6, no.4, pp. 307-321, September 2002.

[3]. T. Camp, J. Boleng, B. Williams, L. Wilcox and W. Navidi. "Performance Comparison of Two Location Based Routing Protocols for Ad Hoc Networks". In Proceedings of IEEE Infocom, pp. 1678- 1687, NY, USAL, June 2002.

[4]. C. Perkins, E. Belding-Royer and S. Das. "Ad hoc On-Demand Distance Vector (AODV) Routing". RFC3561, July 2003.

[5]. J. Li, J. Jannotti, D. S. J. D. Couto, D. R. Karger, and R. Morris. "A Scalable Location Service for Geographic Ad Hoc Routing", in Proceedings of ACM Mobicom 2000, pp. 120-130. Boston, MA, August 2000.

[6]. Z. J. Haas and B. Liang. "Ad Hoc Mobility Management with Uniform Quorum Systems". IEEE/ACM Transactions on Networking, vol. 7, no. 2, pp. 228-240, April 1999.

[7]. S. Lee, B. Bhattacharjee and S. Banerjee. "Efficient geographic routing in multihopwireless networks", in Proceedings of ACM MobiHoc, pp. 230-241, Urbana-Champaign, IL, USA, May 2005.

[8]. Q. Chen, S. S. Kanhere, M. Hassan and K. C. Lan. "Adaptive Position Update in Geographic Routing", in Proceedings of the InternationalConference on Communications (ICC 2006), pp. 4046- 4051, Istanbul, Turkey, June 2006.

[9]. M. Heissenbuttel, T. Braun, M. Walchli and T. Bernoulli. "Evaluating of the limitations and alternatives in beaconing", in Ad Hoc Networks, vol. 5, no. 5, pp. 558-578, 2007.

[10]. M. Heissenbuttel, T. Braun, T. Bernoulli et al. "BLR: Beacon-Less Routing Algorithm for Mobile Ad-Hoc Networks", in Computer Communications, vol,27, pp. 1076-1086, July 2004.

[11]. S. Basagni, M. Nati, C. Petrioli and R. Petroccia"ROME: routing over mobile elements in WSNs", in Proceedings of the 28th IEEE conference on Global telecommunications (GLOBECOM), pp. 5221-5227. Honolulu, Hawaii, USA, December, 2009.

[12]. L. M.Feeney and M. Nilsson. "Investigating the energy consumption of a wireless network interface in an ad hoc networking environment". In IEEE Infocom, pp. 1548-1557,2001. 